\title{
Laboratory capability and surveillance testing for Middle East respiratory syndrome coronavirus infection in the WHO European Region, June 2013
}

\author{
D Pereyaslov (PDM@euro.who.int) ${ }^{1}$, P Rosin², D Palm², H Zeller², D Gross¹, C S Brown¹, M J Struelens ${ }^{2}$, on behalf of the MERS-CoV \\ Working Group ${ }^{3}$ \\ 1. World Health Organization (WHO) Regional Office for Europe, Copenhagen, Denmark \\ 2. European Centre for Disease Prevention and Control (ECDC), Stockholm, Sweden \\ 3. The experts of this group are listed at the end of this article
}

Citation style for this article:

Pereyaslov D, Rosin P, Palm D, Zeller H, Gross D, Brown CS, Struelens MJ, on behalf of the MERS-CoV Working Group. Laboratory capability and surveillance testing for Middle East respiratory syndrome coronavirus infection in the WHO European Region, June 2013 . Euro Surveill. 2014;19(40):pii=20923. Available online: http:// www.eurosurveillance.org/ViewArticle.aspx?Articleld=20923

Article submitted on 9 October 2013 / published on 9 October 2014

Since September 2012, over 90 cases of respiratory disease caused by a novel coronavirus, now named Middle East respiratory syndrome coronavirus (MERS(oV), have been reported in the Middle East and Europe. To ascertain the capabilities and testing experience of national reference laboratories across the World Health Organization (WHO) European Region to detect this virus, the European Centre for Disease Prevention and Control (ECDC) and the WHO Regional Office for Europe conducted a joint survey in November 2012 and a follow-up survey in June 2013. In 2013, 29 of 52 responding WHO European Region countries and 24 of 31 countries of the European Union/European Economic Area (EU/EEA) had laboratory capabilities to detect and confirm MERS-CoV cases, compared with 22 of 46 and 18 of 30 countries, respectively, in 2012. By June 2013, more than 2,300 patients had been tested in 23 countries in the WHO European Region with nine laboratory-confirmed MERS-CoV cases. These data indicate that the Region has developed significant capability to detect this emerging virus in accordance with WHO and ECDC guidance. However, not all countries had developed capabilities, and the needs to do so should be addressed. This includes enhancing collaborations between countries to ensure diagnostic capabilities for surveillance of MERS-CoV infections across the European Region.

\section{Background}

In September 2012, a novel coronavirus was first characterised at the Erasmus Medical Center (EMC), Rotterdam, the Netherlands, by genome sequencing of a viral isolate from a patient in Saudi Arabia with severe pneumonia [1] and was later designated as Middle East respiratory syndrome coronavirus (MERSCoV) [2]. Coronaviruses are enveloped viruses with a positive-sense, single-stranded RNA genome. They can cause respiratory and enteric infections in humans and animals $[3,4]$. Coronaviruses known to infect humans include the human hCoV-229E and hCoV-NL63 alphacoronaviruses, as well as hCoV-OC43, hCoV-HKU1 [5], severe acute respiratory syndrome (SARS)-CoV [6] and now MERS-CoV betacoronaviruses [1]. As of 22 July 2013, there have been 90 laboratory-confirmed cases of human infection with MERS-CoV in the Middle East, North Africa and Europe, including 45 deaths. Of these, nine confirmed cases and five deaths directly or indirectly linked to the Middle East had been reported by four countries in the European Region (France, Germany, Italy and the United Kingdom (UK)) $[7,8]$.

As per the current testing guidance of the World Health Organization (WHO), screening and confirmation of the MERS-CoV infection is based on detection of viral RNA by reverse transcription-polymerase chain reaction (RT-PCR) and sequencing [9]. The testing algorithm includes a two-step approach: (i) screening, targeting the region upstream of the $E$ gene (upE RT-PCR [10],) and (ii) confirmation, targeting the open reading frame 1a (ORF1a RT-PCR [11]). Alternatively, screening and confirmatory testing could be done by targeting other specific regions in the MERS-CoV genome, such as $R d R p$ and/or $N$ genes, and sequence determination of the amplified product $[11,12]$. Surveillance recommendations for human MERS-CoV infections are available from WHO [13] and the European Centre for Disease Prevention and Control (ECDC) [14]; an overview of these and other recommendations for the investigation of MERS-CoV cases is available in Pebody et al. [15]. Any probable or confirmed case should be rapidly reported to national authorities to enable appropriate public health measures. National authorities must notify WHO under the International Heath Regulations (IHR) of any probable and/or confirmed case, and EU/ EEA countries may simultaneously report via the EU/ EEA Early Warning and Response System (EWRS).

Since laboratories are often in the front-line in the detection of emerging pathogens, ECDC jointly with WHO Regional Office for Europe conducted a rapid 
survey in November 2012 to ascertain the capabilities of laboratories across the WHO European Region to detect MERS-CoV [16]. Results showed that 22 of 46 countries in the WHO European Region, including 18 of $30 \mathrm{EU} / \mathrm{EEA}$ countries, had laboratory capability to detect and confirm cases of MERS-CoV. The results also indicated the rapid development of diagnostic capabilities in the responding countries. Since the time of the survey, the epidemiological situation of MERS-CoV has evolved $[7,8]$, including a 10 -fold increase of confirmed cases as well as new travel-related cases and transmission to secondary cases in Europe and elsewhere. Moreover, new diagnostic assays, including molecular and serological assays have been developed $[11,17,18]$.

To determine the current level of MERS-CoV diagnostic capabilities in the Region and assess the recent testing practices in relation to national and international surveillance guidance, ECDC and WHO Regional Office for Europe initiated a follow-up survey in June 2013. The results of this survey are presented here.

\section{Survey of MERS-CoV detection and confirmation capabilities}

The survey covered the following four areas: (i) availability of laboratory tests for detection and characterisation of MERS-CoV from human specimens; (ii) criteria used for laboratory testing and case ascertainment in relation to national, EU and international guidance; (iii) testing experience and outcome to date per country; and (iv) needs for laboratory support from ECDC and/ or WHO.

The survey was administered to all countries in the WHO European Region including 53 Member States and two States Parties to the IHR (Liechtenstein and the Holy See), including the 31 EU/EEA countries. The ECDC sent the survey request by email to the National Microbiology Focal Points of the EU/EEA countries and to contact points for laboratories in the European Network for Diagnostics of 'Imported' Viral Diseases (ENIVD). The WHO Regional Office sent the survey by email to EuroFlu National Focal Points from National Influenza Centres (NICS) in non-EU/EEA countries and institutions responsible for MERS-CoV testing identified during the first survey.

\section{FIGURE 1}

Progressive implementation of laboratory tests for detection and confirmation of Middle East respiratory syndrome coronavirus in the World Health Organization European Region, by country, November 2012-June 2013 (n=52)

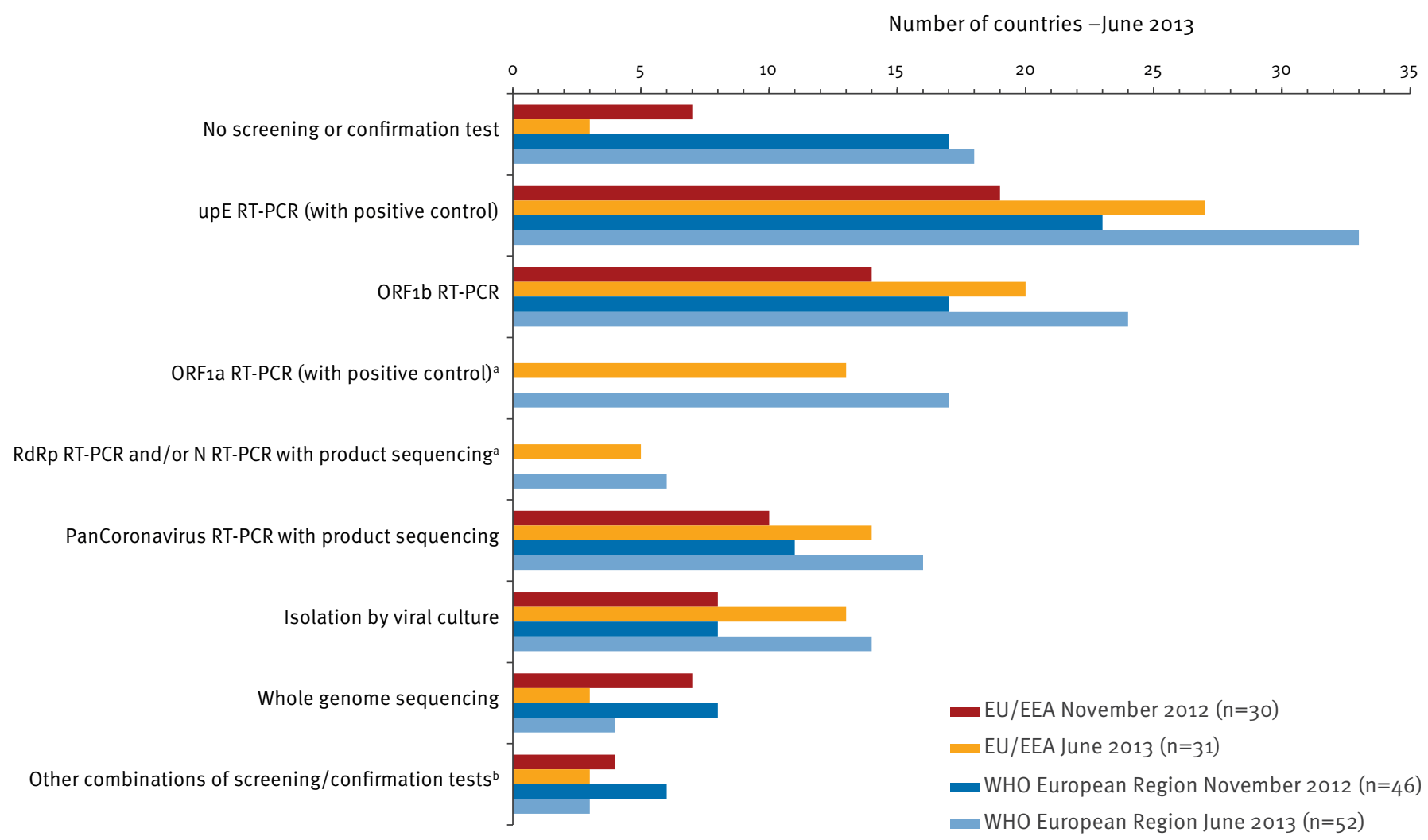

EU/EEA: European Union/European Economic Area; RdRP: RNA-dependent RNA polymerase; RT-PCR: reverse transcription-poymerase chain reaction; WHO: World Health Organization.

a Data for November 2012 when these tests were not yet developed.

b Other combinations of screening/confirmation tests include in-house-developed assays as well as the use of commercially available RT-PCR kits for human coronaviruses. 
Data were collected until 28 June 2013 and were validated by the survey respondents on 5 August 2013.

\section{Survey results}

The survey captured data from 52 of 55 countries in the WHO European Region including all 31 EU/EEA countries, giving response rates of $94 \%$ and $100 \%$, respectively. The survey captured capabilities of 72 laboratories in the Region, of which 36 laboratories from 34 countries were WHO-recognised NICs and 30 laboratories from 21 countries were partners of ENIVD.

\section{Availability of assays for MERS-CoV laboratory testing}

Data showed that 33 of 52 countries in the WHO European Region and 27 of 31 EU/EEA countries had implemented upE RT-PCR screening tests with a positive control. This assay was available in 51 laboratories in the WHO European Region and 41 laboratories in the EU/EEA countries. The most frequently implemented confirmation test was RT-PCR for ORF1b, which was available in 24 of 52 countries in the WHO European Region and 20 of 31 EU/EEA countries. Confirmation using RT-PCR targeting ORF1a was available in 17 of 52 and 13 of 31 countries, respectively. Application of $R d R p$ and/or $N$ gene RT-PCR followed by sequence determination was confirmed by six of 52 and five of 31 countries, respectively. Five of the responding countries in the WHO European Region indicated that they had serological assays for MERS-CoV testing available. These tests included IgG and IgM immunofluorescence assay, Western blot against recombinant $\mathrm{N}$ protein, serum neutralisation tests $[11,17]$, or protein microarray using in-vitro expressed coronavirus spike proteins as antigens [18]. Figure 1 shows the progressive implementation of screening/confirmation tests between November 2012 and June 2013 for MERS-CoV in the responding countries.

Based on the information on available tests and using the WHO interim case definition [12], we analysed the different degrees of MERS-CoV diagnostic capabilities in the region (Figure 2). We found that 29 of 52 countries in the Region and 24 of 31 EU/EEA countries had the capability to screen and confirm human MERS-CoV cases, compared with 22 of 46 and 18 of 30 respectively, reported in the November 2012 assessment [16]. Only screening using upE RT-PCR was available in five and four of the responding countries in the Region and EU/EEA, respectively. Seventeen countries had no national-level capability for MERS-CoV detection and confirmation; most were located in the south-eastern

\section{FIGURE 2}

Laboratory capabilities for detection and confirmation of Middle East respiratory syndrome coronavirus in the World Health Organization European Region, by country, June 2013 ( $\mathrm{n}=52)$

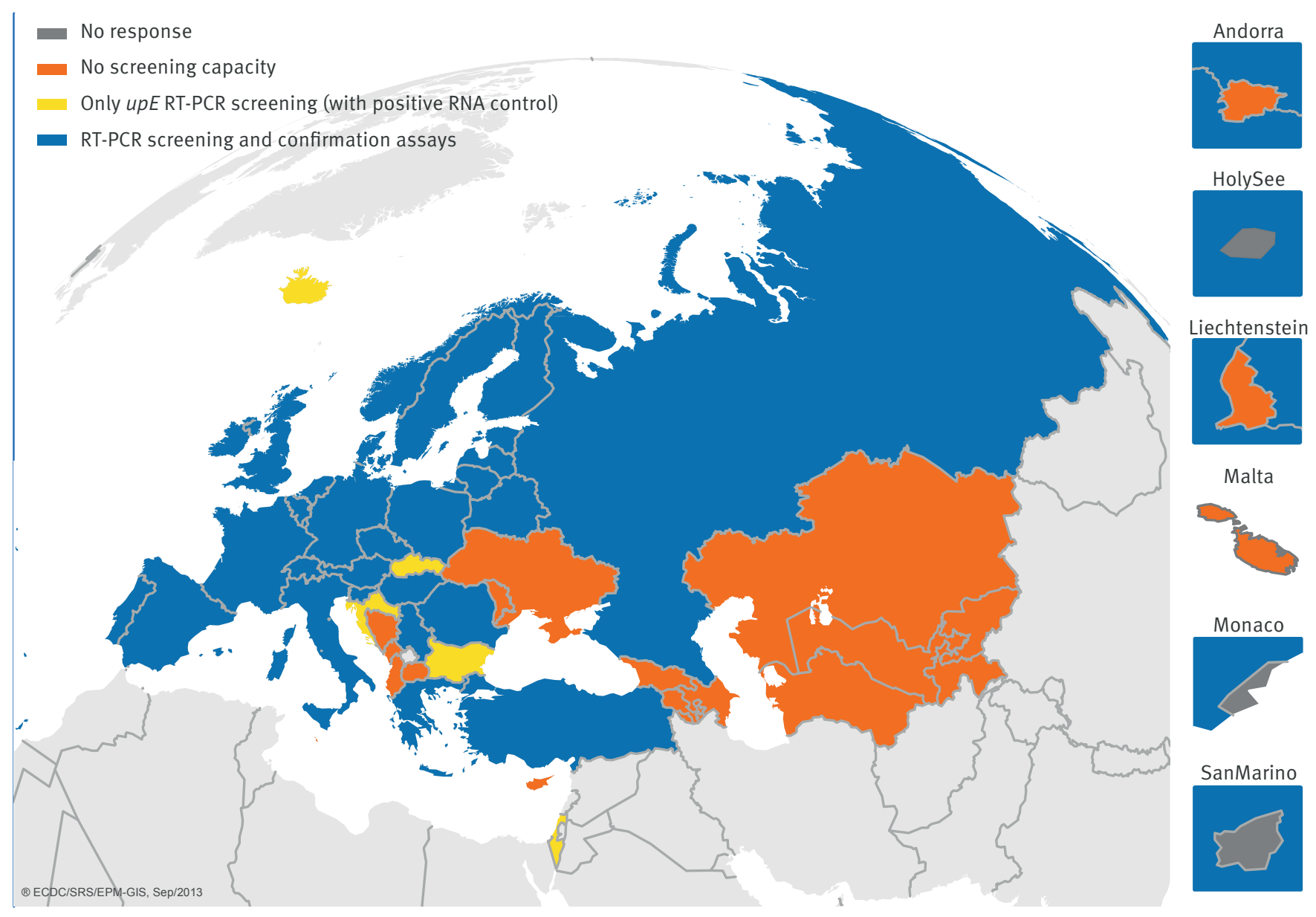


and eastern European part of the Region. All three EU/ EEA countries without MERS-CoV screening/confirmation capability reported that referral arrangements were in place for shipment of specimens to other laboratories in the EU.

Recommendations used for testing and testing experience by indication and type of specimens The recommendations for MERS-CoV testing that were reported as being applied at national level were the WHO interim surveillance recommendations [13], followed by 36 of 52 countries in the Region and 23 of 31 EU/EEA countries, and the ECDC surveillance recommendations [14], used in 22 of 52 and 18 of 31 countries, respectively. Other testing guidance documents used were issued by national authorities ( 16 of 52 and 10 of 31 countries, respectively) and two countries followed the recommendations of the United States Centers for Disease Control and Prevention.

Following the respective recommendations, between the period of September 2012 and June 2013, 23 of 52 countries in the Region and 19 of 31 EU/EEA countries reported testing of human samples for MERS-CoV. The number of patients tested varied by country as indicated in Figure 3 and Table 1. Note that the relatively high numbers of patients tested in Belgium and Italy were due to screening for MERS-CoV being included in routine testing as part of surveillance for severe acute respiratory infections (SARI). The majority of countries with testing experience tested between one and 10 patients during the studied period ( 13 of 23 countries in the Region and 11 of 19 EU/EEA).

Nearly $80 \%$ of all samples tested were specimens from the upper respiratory tract (Table 1). Specimens from the lower respiratory tract were used in $17 \%$ of all samples tested. Other types of specimens reported included urine and serum samples.

Excluding 1,812 patients from Belgium and Italy tested for MERS-CoV as part of the routine surveillance scheme for SARI, testing of 522 patients in the 23 countries followed the indications recommended for surveillance. In 367 cases, one of the main reasons reported for triggering testing was the symptoms exhibited by the patient. Recent travel to the Middle East in patients with pneumonia or acute respiratory distress syndrome (ARDS) was reported as a reason for testing in 319 cases, and developing of respiratory symptoms following close contact with a confirmed or probable case of MERS-CoV infection in 114 cases. Other reasons for

\section{FIGURE 3}

Number of patients tested for Middle East respiratory syndrome coronavirus in the World Health Organization European Region, by country, September 2012-June $2013(\mathrm{n}=52)$
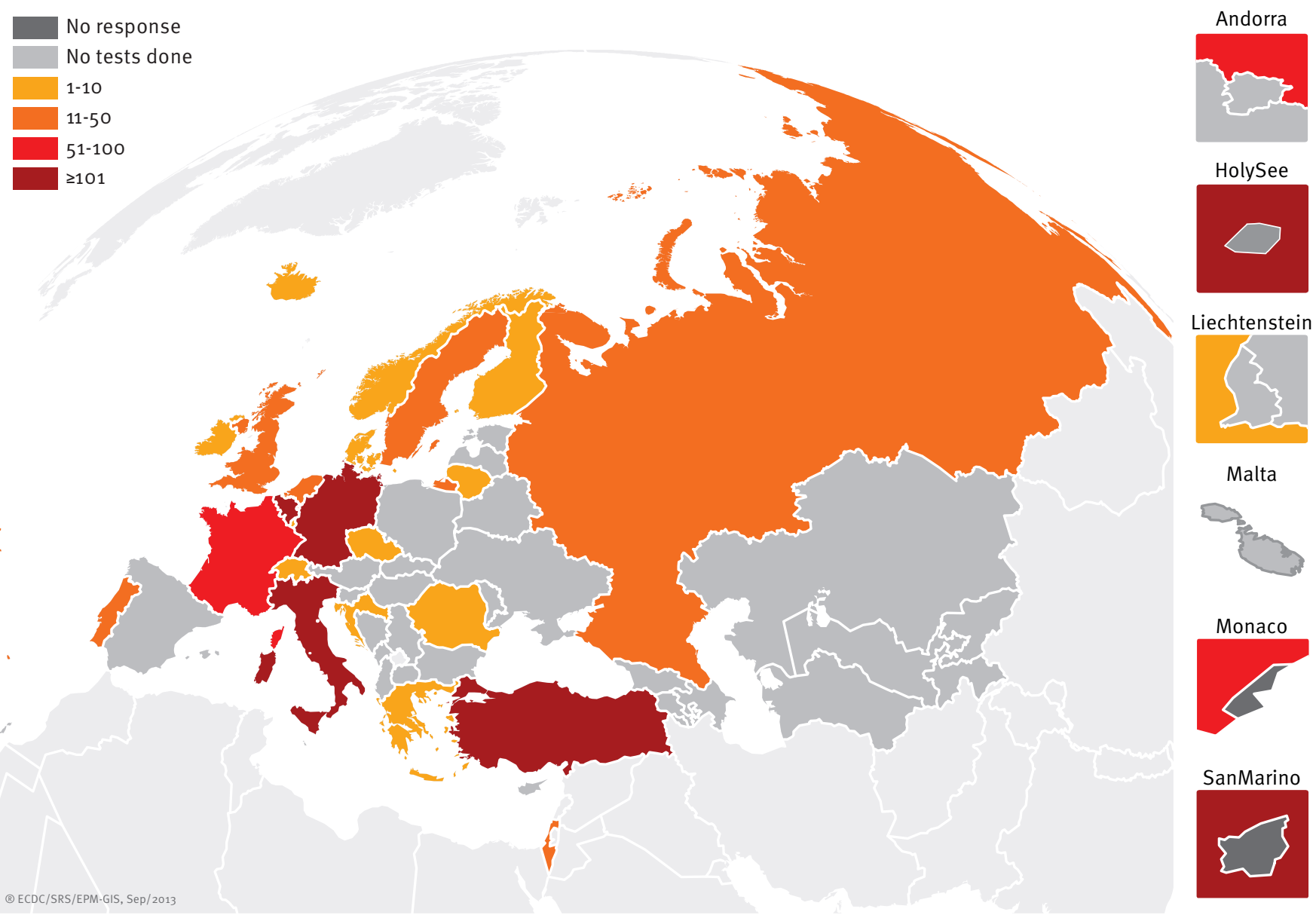


\section{TABLE 1}

Testing experience for Middle East respiratory syndrome coronavirus in the World Health Organization European Region, per country, June $2013(\mathrm{n}=52)$

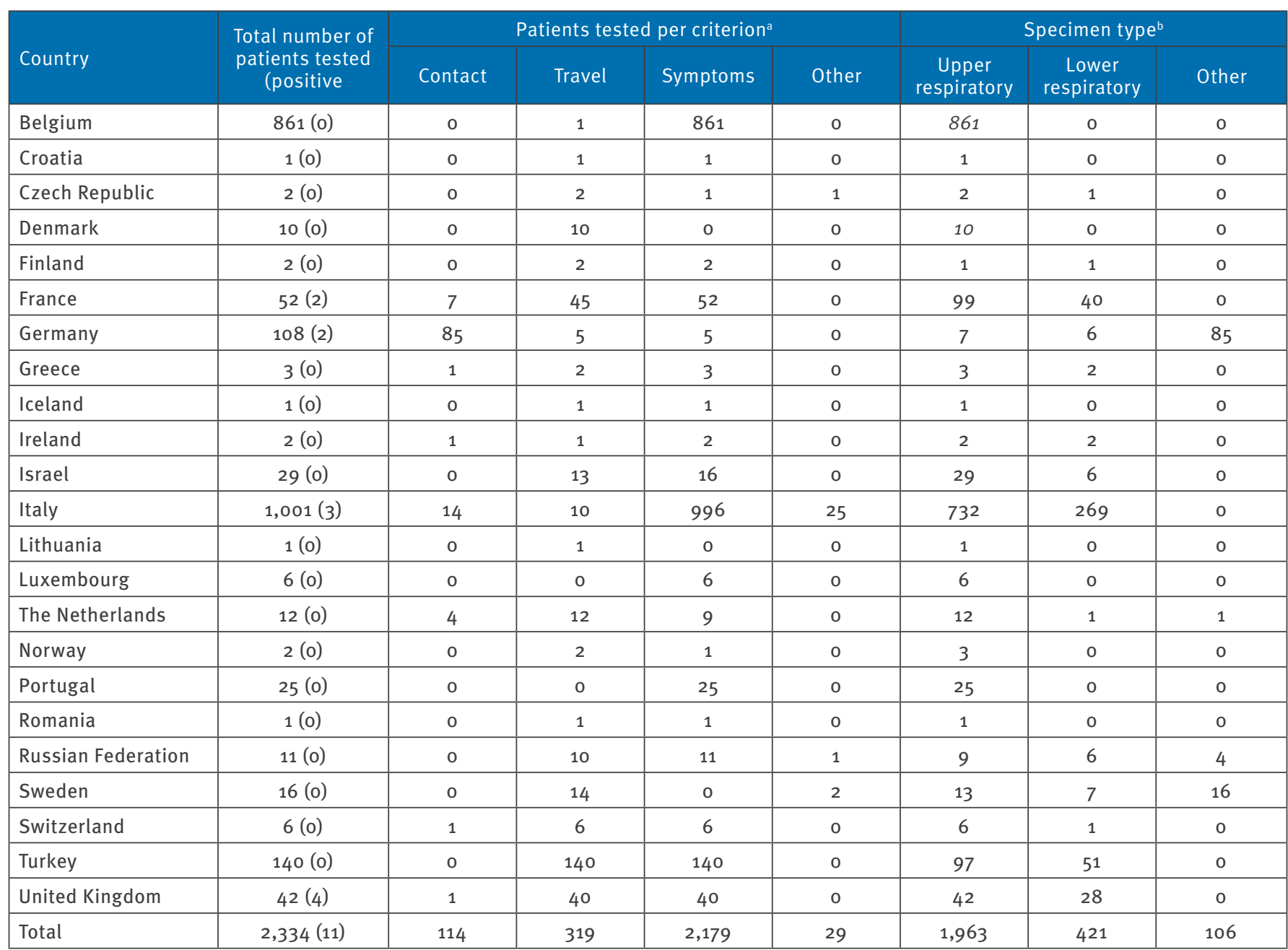

a Criterion 'Contact' refers to close physical contact with a confirmed or probable MERS-CoV case; 'Travel' refers to travel to the Arabian peninsula or neighbouring countries within 10 to 14 days before onset of illness; 'Symptoms' refers to febrile acute respiratory illness with clinical, radiological, or histopathological evidence of pulmonary parenchymal disease (e.g. pneumonia or acute respiratory distress syndrome).

b Multiple specimens were collected for some patients.

\section{TABLE 2}

Needs for laboratory support for Middle East respiratory syndrome coronavirus detection and confirmation in the World Health Organization European Region, by country, June 2013 ( $\mathrm{n}=52)$

\begin{tabular}{|c|c|c|}
\hline Area of support & EU/EEA $(n=31)$ & $\begin{array}{l}\text { WHO European Region } \\
\qquad(\mathrm{n}=52)\end{array}$ \\
\hline No support needed & 11 & 13 \\
\hline Provision of primers and probes & 8 & 23 \\
\hline Provision of positive control material for RT-PCR & 15 & 32 \\
\hline Assistance with shipment abroad for MERS-CoV testing & 7 & 16 \\
\hline Other type of support & 8 & 12 \\
\hline
\end{tabular}

EU/EEA: European Union/European Economic Area; MERS-CoV: Middle East respiratory syndrome coronavirus; WHO: World Health Organization. 
testing included patients in intensive care with severe acute respiratory infections for which no other causative infectious agents were detected. More than 300 patients tested for MERS Co-V had fulfilled at least two of the criteria for testing. Countries that performed the largest part of testing were Turkey, Germany, France and the UK.

\section{Need for laboratory support from ECDC and/ or WHO}

When asked about laboratory support needed, $75 \%$ of the reporting countries in the Region and $65 \%$ of those in the EU/EEA identified needs for laboratory diagnostic support from ECDC and/or WHO (Table 2). The most frequently stated need was continued provision of positive control material for RT-PCR $(63 \%$ countries in the Region and $48 \%$ of EU/EEA countries). Other needs included training of laboratory personnel, provision of RT-PCR reagents and consumables, assistance with viral culture and serological assays.

\section{Discussion}

The findings of this study show that 10 months after sequence information for the first reported MERS-CoV case was made available [1], 29 of 52 countries in the WHO European Region and 24 of 31 EU/EEA countries have developed laboratory capabilities to detect and confirm MERS-CoV cases. Compared with the assessment of these capabilities in November 2012 [16], an additional seven countries in the Region and six in the EU/EEA had implemented MERS-CoV detection and confirmation capability by June 2013. While case confirmation was done mainly by ORF1b RT-PCR and whole genome sequencing in November 2012 [16], several additional specific assays are now in use in Europe's expert microbiology laboratories (RT-PCR for ORF1a, RdRp and the $N$ gene, followed by sequencing). Moreover, 23 laboratories in 14 countries in the Region are now capable of isolating and identifying MERSCoV by culture, compared with 16 laboratories in eight countries in November 2012. Interestingly, the number of countries using other combinations of screening/ confirmation tests as well as whole genome sequencing has decreased as international testing recommendations has become available and commonly accepted methods for screening and confirmation have been implemented.

The rapid increase in diagnostic capabilities described here is due to dedicated efforts at national level, to support from WHO via its network of NICs and from ECDC via dedicated laboratory networks (e.g. ENIVD) and to other EU initiatives. The European Virology Archive (EVA) for example, allowed laboratories to receive positive control material for the upE and ORF1a RT-PCR assays to set up the necessary MERS-CoV diagnostic assays.

However, a large proportion of laboratories still need support for the provision of positive control material as well as primers and probes for RT-PCR, and assistance with shipment abroad for MERS-CoV testing. These remaining needs are of concern, especially for countries neighbouring MERS-CoV endemic areas. Therefore, WHO is currently analysing the factors hampering the uptake of MERS-CoV diagnostic assays in this part of the Region. The aim of this analysis is to set up a mechanism which ensures Region-wide deployment of laboratory diagnostic assays for MERS-CoV.

ECDC is currently supporting an external quality assessment scheme for MERS-CoV via ENIVD, addressing laboratory performance and pending gaps in capabilities for detection and confirmation of MERS-COV in the Region. Thus, building on existing WHO and EU laboratory networks, the two agencies will strive to maintain and further enhance diagnostic capabilities for MERS-CoV.

Our survey collected information on the types of clinical specimens used for testing. However, we did not collect specific information on what proportion of specimens were used for MERS-CoV diagnosis vs follow-up of diagnosed patients or monitoring of viral loads. The majority of specimens used for MERS-CoV testing were obtained from the upper respiratory tract which, according to preliminary reports, may contain lower viral loads than specimens from the lower respiratory tract $[19,20]$. Therefore, it is advisable to increase awareness among healthcare providers of the benefits of obtaining specimens from the lower respiratory tract when possible, particularly in case of disease progression, and to integrate this recommendation into national laboratory testing algorithms.

Importantly, the improved capabilities for MERS-CoV case confirmation were accompanied by increased testing in the European Region: since September 2012, over 2,300 patients have been tested in 23 countries. Apart from two countries that extended MERS-CoV detection tests to SARI patients irrespective of travel or contact history, the vast majority of countries focused on travellers with pneumonia or ARDS upon recent return from the Middle East and patients with close contact with a confirmed or probable case of MERS$\mathrm{CoV}$ infection, in compliance with international guidance. However, the number of patients fulfilling these clinical and epidemiological criteria during the study period was not collected and we can therefore not estimate the case-finding bias per country and across the Region as a whole. In September 2013, WHO published updated recommendations for laboratory testing of MERS-CoV [9]. These recommendations highlight the need for intensified efforts to validate serological tests for case finding and serological studies in risk groups and targeted populations. At the time of our study, existing serological tests had been validated against small numbers of convalescent sera, and there is no consensus on the interpretations of the results. WHO, ECDC and their networks will investigate possibilities to enhance the collaboration with countries in affected regions which would provide a platform for validating 
serological assays. At the time of this study, only five countries indicated that they had the capability to perform assays for MERS-CoV-specific antibody detection.

After submission of this manuscript, three countries in the Region reported cases of MERS-CoV in returning travellers or residents of countries of the Middle East: Greece (one case, April 2014), the Netherlands (two cases, May 2014) and Austria (one case, September 2014). In total 14 laboratory-confirmed cases have been reported since April 2012. More information is available in the updated ECDC rapid risk assessment from August 2014 and the epidemiological update from October 2014 [21,22].

In order to provide laboratories with the opportunity to assess their capabilities, a first external quality assurance (EQA) panel for the detection of MERS-CoV by PCR was organised in spring 2014 by ENIVD with support of ECDC and WHO. Laboratories in 33 countries the Region participated in this scheme. A feasibility of a new global EQA scheme is currently being explored by WHO. A training for national public health institutes on laboratory preparedness and rapid establishment of detection assays for emerging respiratory pathogens will be conducted in November 2014. Based on the new evidence on MERS-CoV infection and new information on diagnostic assays, WHO issued updates of surveillance, case definition and laboratory recommendations $[23,24,25]$. The major change compared with the previous version is that a patient may be considered as a confirmed case if a four-fold rise in neutralising antibody titre can be demonstrated, regardless of any PCR results.

\section{Conclusion}

The decision taken at the second meeting of the Emergency Committee convened by the WHO DirectorGeneral on 17 July 2013 under the IHR was that the current outbreak of MERS-CoV is not a Public Health Emergency of International Concern (PHEIC) [26]. Importantly however, it was noted that while the PHEIC conditions had not been met, WHO, ECDC and Member States should continue to be vigilant in their surveillance for MERS-CoV. Although only fourteen confirmed MERS-CoV cases have been identified in the Region since April 2012, the substantial amount of testing reported here serves as a reassurance of the existing laboratory support to MERS-CoV surveillance. As the present study shows, there is robust capability for detection and confirmation of human MERS-CoV cases in the EU/EEA. However, one third of the countries of the WHO European Region, mainly in the south-east and eastern part of the Region, are still lacking MERSCoV diagnostic capabilities. Therefore, efforts continue to address the remaining laboratory needs in order to ensure MERS-CoV detection and confirmation capability needed for active surveillance of this emerging disease in Europe.
Experts of the MERS-CoV Working Group

Members of this working group who provided survey data: Albania: Alma Robo, Iris Hasibra (Hatibi), Institute of Public Health, Tirana

Andorra: Josep Casals Alis, Ministry of Health, Welfare and Labour, Andorra la Vella

Armenia: Shushan Sargsyan, Virology Laboratory, Centre for Diseases Control and Prevention, Yerevan

Austria: Stephan Aberle, Department of Virology, Medical University of Vienna, Vienna

Azerbaijan: Sadraddin Gurbanov, National Virology Laboratory, National Anti-Plague Station, Baku

Belarus: Natalia Gribkova, Laboratory for Influenza and Influenza-like Diseases, Republican Research and Practical Center for Epidemiology and Microbiology, Minsk

Belgium: Marc Van Ranst, Greet leven and Sophie Patteet, National Reference Centre of Respiratory Viruses, University Hospital Leuven and UZA Antwerpen, Antwerpen

Bosnia and Herzegovina: Stanka Tomic, Microbiology Department, Institute of Public Health of the Republic of Srpska, Banja Luka

Bulgaria: Neli Korsun, National Laboratory "Influenza and ARD”, Department of Virology, National Centre of Infectious and Parasitic Diseases, Sofia

Croatia: Vladimir Drazenovic, National Influenza Centre, Croatian National Institute of Public Health, Zagreb

Cyprus: Despo Pieridou-Bagkatzouni, Microbiology Department, Nicosia General Hospital, Nicosia

Czech Republic: Helena Jirincova, Martina Havlickova, National Reference Laboratory for Influenza, National Institute for Public Health, Prague

Denmark: Anders Fomsgaard, Virus Research and Development Laboratory, Department Microbiology Diagnostic and Virology, Statens Serum Institut, Copenhagen Estonia: Külli Rae, Laboratory of Communicable Diseases, Health Board, Tallinn

Finland: Maija Lappalainen, Department of Virology and Immunology, Helsinki University Hospital, Laboratory Services (HUSLAB) and Niina Ikonen, Virology Unit, National Institute for Health and Welfare, Helsinki

France: Bruno Lina, Centre National de Référence des Virus Influenza - HCL, Lyon and Sylvie van der Werf, Unit of Molecular Genetics of RNA viruses, Institut Pasteur and Jean-Claude Manuguerra, Cellule d'Intervention Biologique d'Urgence (CIBU), Institut Pasteur, Paris

Georgia: Ann Machablishvili, National Influenza Centre, National Centre for Disease Control and Public Health, Tbilisi Germany: Markus Eickmann, Institut für Virologie der Philipps-Universität in Marburg and Thorsten Wolff, Div of Influenza and other Respiratory viruses; Robert KochInstitut, and Dr. Gerhard Dobler, Bundeswehr Instittue of Microbiology, and Jonas Schmidt-Chanasit, WHOCC for Arbovirus and Haemorrhagic Fever Reference and Research at Bernhard Nocht Institute for Tropical Medicine, Hamburg, and Christian Drosten, Virology Institute, Bonn

Greece: Anna Papa, National Reference Laboratory for Arboviruses and Hemorrhagic Fever viruses, Aristotle University of Thessaloniki, Thessaloniki and Andreas F. Mentis, National Influenza Reference Laboratory of Southern Greece/Hellenic Pasteur Institute, Athens

Hungary: Zoltan Kis, Department for Respiratory Viruses / National Biosafety Laboratory, B. Johan National Center for Epidemiology, Budapest

Iceland: Arthur Löve, Department of Virology, LandspitaliNational University Hospital, Reykjavik

Ireland: Suzie Coughlan, National Virus Reference Laboratory/University College Dublin, Dublin

Israel: Michal Mandelboim, Central Virology Laboratory, Sheba Medical Center, Tel Hashomer

Italy: Maria R. Capobianchi, Laboratory of Virology/National Institute for Infectious Diseases Lazzaro Spallanzani, and Maria Paola Landini, Regional Center for Emerging Infections (CRREM)/ Unit of Clinical Microbiology, St. Orsola General 
Hospital, Bologna, and Fausto Baldanti, Molecular Virology Unit, Department of Microbiology and Virology, Fondazione IRCCS Policlinico San Matteo, Pavia, and Giorgio Palu, Microbiology and Virology/Padova University Hospital, and Valeria Ghisetti, Laboratory of Microbiology and Virology, Amedeo di Savoia Hospital, Torino, and Isabella Donatelli, National Influenza Centre, Instituto Superiore di Sanita,

Kazakhstan: Gaukhar Nusupbayeva, Zarina Tokhtabakiyeva, National Reference Laboratory on Control of Viral Infections, Scientifical-Practical Center of Sanitary and Epidemiological Expertise and Monitoring, Almaty

Kyrgyzstan: Kaliya Kasymbekova, Centre of MolecularGenetic and Microbiological Investigations, Department of State Sanitary Epidemiological Surveillance, Bishkek

Latvia: Jelena Storozenko, Riga East University Hospital, Latvian Centre of Infectious Diseases, National Microbiology Reference Laboratory, Riga

Liechtenstein: Sabine Erne, Office of Public Health, Country Administration of Principality of Liechtenstein

Lithuania: Algirdas Griskevicius, National Public Health Surveillance Laboratory, Vilnius

Luxembourg: Matthias Opp, Laboratoire National de Santé, Luxembourg

Malta: Christopher Barbara, Pathology Department, Mater Dei Hospital, Msida

Montenegro: Zoran Vratnica, Centre for Medical Microbiology, Public Health Institute of Montenegro, Podgorica

Netherlands: Chantal Reusken, Centre for Infectious Disease Research, Diagnostics and Screening, National Institute for Public Health and the Environment, Bilthoven

Norway: Susanne Gjeruldsen Dudman and Olav Hungnes, Department of Virology, Norwegian Institute of Public Health, Oslo

Poland: Katarzyna Pancer, National Institute of Public HealthNational Institute of Hygiene, Department of Virology, Warsaw

Portugal: Raquel Guiomar, National Influenza Reference Laboratory, Infectious Diseases Department, National Institute of Health, Lisboa

Republic of Moldova: Veronica Eder, Laboratory of Viral Respiratory Infections, National Center for Public Health, Chisinau

Romania: Emilia Lupulescu, Laboratory for Respiratory Viruses/ NIRDMI Cantacuzino, Bucharest

Russian Federation: Svetlana Yatsyshina, Reference Centre for Infection Agents, Central Research Institute of Epidemiology (CRIE), Rospotrebnadzor, Moscow, and Maria Pisareva and Zhanna Buzitskaya, Laboratory of Molecular Virology and Genetic Engineering, Research Institute of Influenza, St Petersburg, and Alexander Sergeev, State Research Center of Virology and Biotechnology VECTOR, Novosibirsk

Serbia: Jasminka Nedeljković, Respiratory Department, Torlak Institute of Immunology and Virology, Belgrade

Slovakia: Edita Staroňová, National Influenza Center/Public Health Authority, Bratislava

Slovenia: Tatjana Avšič Županc, Miroslav Petrovec, Miša Korva, University of Ljubljana, Faculty of Medicine, Institute of Microbiology and Immunology, and Katarina Prosenc, Laboratory for Virology, National Public Health Institute Slovenia, Ljubljana

Spain: Inmaculada Casas, Influenza National Reference Laboratory, National Influenza Center-Madrid, Instituto de Salud Carlos III, Majadahonda, Madrid and Ramon Cisterna Clinical microbiology and infection control, Hospital Basurto Bilbao Spain

Sweden: Hans Gaines, Swedish Institute for Communicable Disease Control, Stockholm

Switzerland: Pascal Cherpillod, National Reference Centre for Emerging Viral Infections, Laboratory of Virology, Division of Infectious Diseases University of Geneva Hospitals, Geneva Tajikistan: Niginamo Zakirova, Virology Laboratory, State Sanitary-Epidemiological Surveillance, Dushanbe
The former Yugoslav Republic of Macedonia: Golubinka Bosevska, Laboratory for Virology and Molecular Diagnostics, Institute of Public Health, Skopje

Turkey: Basak Altas, National Influenza Centre, Virology Reference and Research Laboratory, Public Health Institutions of Turkey, Ankara, and Meral Ciblak, National Influenza Reference Laboratory, Faculty of Medicine, University of Istanbul, Istanbul

Turkmenistan: Central Reference Laboratory, Sanitary Epidemiologic Service, Ashgabat

Ukraine: Alla Mironenko, National Influenza Centre, L.V.Gromashevsky Institute of Epidemiology \& Infectious diseases NAMS, and Tetiana Dykhanovska and Iryna Demchyshyna, Centre of influenza and ARVI, Central Sanitary and Epidemiological Station, Kiev

United Kingdom: Alison Bermingham, Respiratory Virus Unit, Virus Reference Department, Public Health England, London Uzbekistan: Ravshan Rakhimov, National Influenza Centre, Institute of Virology, Tashkent.

\section{Acknowledgements}

We thank the ECDC National Microbiology Focal Points in EU/ EEA countries, focal points from laboratories of the EuroFlu and ENIVD networks for coordinating data collection and for dedicated and rapid responses to the surveys.

\section{Conflict of interest}

None declared.

Authors' contributions

None declared.

\section{Disclaimer}

The authors alone are responsible for the views expressed in this article and they do not necessarily represent the views, decisions or policies of the institutions with which they are affiliated. Maps used in this paper do not imply any opinions on the part of ECDC and WHO or its partners about the legal status of the countries and territories shown or about their borders.

\section{References}

1. van Boheemen S, de Graaf M, Lauber C, Bestebroer TM, Raj VS, Zaki AM, et al. Genomic characterization of a newly discovered coronavirus associated with acute respiratory distress syndrome in humans. MBio. 2012;3(6):pii=e00473-12. http:// dx.doi.org/10.1128/mBio.00473-12. PMID:23170002

2. de Groot RJ, Baker SC, Baric RS, Brown CS, Drosten C, Enjuanes $\mathrm{L}$, et al. Middle East respiratory syndrome coronavirus (MERS (oV): announcement of the Coronavirus Study Group. J Virol. 2013;87(14):7790-2. http://dx.doi.org/10.1128/JVI.01244-13. PMID:23678167

3. Weiss SR, Leibowitz JL. Coronavirus pathogenesis. Adv Virus Res. 2011;81:85-164. http://dx.doi.org/10.1016/B978-0-12385885-6.00009-2. PMID:22094080

4. Bolles M, Donaldson E, Baric R. SARS-CoV and emergent coronaviruses: viral determinants of interspecies transmission. Curr Opin Virol. 2011;1(6):624-34. http://dx.doi.org/10.1016/j. coviro.2011.10.012. PMID:22180768

5. van der Hoek L. Human coronaviruses: what do they cause? Antivir Ther. 2007;12(4 Pt B):651-8. PMID:17944272

6. Lau YL, Peiris JS. Pathogenesis of severe acute respiratory syndrome. Curr Opin Immunol. 2005;17(4):404-10. http:// dx.doi.org/10.1016/j.coi.2005.05.009. PMID:15950449

7. World Health Organization (WHO). Global alert and response. Middle East respiratory syndrome coronavirus (MERS-CoV) 
- update. Geneva: WHO; 21 July 2013. Available from: http:// www.who.int/csr/don/2013_07_21/en/index.html

8. European Centre for Disease Prevention and Control (ECDC). Rapid risk assessment: Severe respiratory disease associated with Middle East respiratory syndrome coronavirus (MERSCoV). Stockholm: ECDC; 22 July 2013. Available from: http:// www.ecdc.europa.eu/en/publications/Publications/RRA-ECDCMERS-CoV-Sixth-update.pdf

9. World Health Organization (WHO). Laboratory testing for Middle East respiratory syndrome coronavirus. Interim recommendations. Geneva: WHO; September 2013 Available from: http://www.who.int/csr/disease/coronavirus_infections/ MERS_Lab_recos_16_Sept_2013.pdf

10. Corman VM, Eckerle I, Bleicker T, Zaki A, Landt O, Eschbach Bludau M, et al. Detection of a novel human coronavirus by real-time reverse-transcription polymerase chain reaction. Euro Surveill. 2012;17(39):pii=20285. PMID:23041020

11. Corman VM, Müller MA, Costabel U, Timm J, Binger T, Meyer B, et al. Assays for laboratory confirmation of novel human coronavirus (hCoV-EMC) infections. Euro Surveill. 2012;17(49): $\mathrm{pii}=20334$. PMID:23231891

12. World Health Organization (WHO). Revised interim case definition for reporting to WHO - Middle East respiratory syndrome coronavirus (MERS-CoV). Geneva: WHO; 3 July 2013. Available from: http://www.who.int/csr/disease/coronavirus infections/case_definition/en/index.html

13. World Health Organization (WHO). Interim surveillance recommendations for human infection with novel coronavirus -update. Geneva: WHO; 18 May 2013. Available from: http://www.who.int/csr/disease/coronavirus_infections/ InterimRevisedSurveillanceRecommendations_ nCoVinfection_18May13.pdf

14. European Centre for Disease Prevention and Control (ECDC). Rapid risk assessment: Severe respiratory disease associated with Middle East respiratory syndrome coronavirus (MERSCoV). Stockholm: ECDC; 17 May 2013. Available from: http:// ecdc.europa.eu/en/publications/Publications/risk-assessmentmiddle-east-respiratory-syndrome-coronavirus-MERS-CoV-17may-2013.pdf

15. Pebody RG, Nicoll A, Buchholz U, Zambon M, Mounts A. Enhanced surveillance and investigation of coronavirus: what is required? East Mediterr Health J. 2013;19(Suppl 1):S55-60. PMID:23888796

16. Palm D, Pereyaslov D, Vaz I, Broberg E, Zeller H, Gross D, et al.; Joint ECDC-WHO Regional Office for Europe Novel Coronavirus Laboratory Survey participants; ECDC National Microbiology Focal Points; WHO European Region EuroFlu Network; European Network for Diagnostics of "Imported" Viral Diseases (ENIVD). Laboratory capability for molecular detection and confirmation of novel coronavirus in Europe, November 2012. Euro Surveill. 2012;17(49): pii=20335. PMID:23231892

17. Buchholz U, Müller MA, Nitsche A, Sanewski A, Wevering N, Bauer-Balci T, et al. Contact investigation of a case of human novel coronavirus infection treated in a German hospital, October-November 2012. Euro Surveill. 2013;18(8): pii $=20406$. PMID:23449231

18. Reusken C, Mou H, Godeke GJ, van der Hoek L, Meyer B, Müller $M A$, et al. Specific serology for emerging human coronaviruses by protein microarray. Euro Surveill. 2013;18(14):20441. http://dx.doi.org/10.2807/1560-7917.ES2013.18.14.20441. PMID:23594517

19. Drosten C, Seilmaier M, Corman VM, Hartmann W, Scheible $\mathrm{G}$, Sack S, et al. Clinical features and virological analysis of a case of Middle East respiratory syndrome coronavirus infection. Lancet Infect Dis. 2013;13(9):745-51. http://dx.doi. org/10.1016/S1473-3099(13)70154-3. PMID:23782859

20. Guery B, Poissy J, el Mansouf L, Séjourné C, Ettahar N, Lemaire $X$, et al.; MERS-CoV study group. Clinical features and viral diagnosis of two cases of infection with Middle East Respiratory Syndrome coronavirus: a report of nosocomial transmission. Lancet. 2013;381(9885):2265-72. http://dx.doi. org/10.1016/S0140-6736(13)60982-4. PMID:23727167

21. European Centre for Disease Prevention and Control (ECDC). Severe respiratory disease associated with Middle East respiratory syndrome coronavirus (MERS-CoV). Updated rapid risk assessment. Stockholm: ECDC; 21 August 2014. Available from: http://www.ecdc.europa.eu/en/publications/ Publications/Middle-East-respiratory-syndrome-coronavirusSaudi\%20Arabia-Qatar-Jordan-Germany-United-Kingdom.pdf

22. European Centre for Disease Prevention and Control (ECDC). Epidemiological update: Middle East respiratory syndrome coronavirus (MERS-CoV). Stockholm: ECDC; 1 October 2014. Available from: http://www.ecdc.europa. eu/en/press/news/_layouts/forms/News_DispForm. aspx? List $=8 \mathrm{db} 7286 \mathrm{c}-\mathrm{fe} 2 \mathrm{~d}-476 \mathrm{c}-9133-18 \mathrm{ff}_{4} \mathrm{cb} 1 \mathrm{~b}_{5} 68 \& \mathrm{ID}=1074$
23. World Health Organization (WHO). Laboratory testing for Middle East respiratory syndrome coronavirus. Interim recommendations (revised). Geneva: WHO; September 2014. Available from: http://www.who.int/csr/disease/coronavirus_ infections/WHO_interim_recommendations_lab_detection_ MERSCoV_092014.pdf?ua =1

24. World Health Organization (WHO). Interim surveillance recommendations for human infection with Middle East respiratory syndrome coronavirus. Geneva: WHO. 14 July 2014. Available from: http://www.who.int/csr/disease/coronavirus infections/InterimRevisedSurveillanceRecommendations_ nCoVinfection_14July2014.pdf?ua=1

25. World Health Organization (WHO). Revised interim case definition for reporting to WHO - Middle East respiratory syndrome coronavirus (MERS-CoV). Geneva: WHO; 14 July 2014. Available from: http://www.who.int/csr/disease/ coronavirus_infections/case_definition/en/

26. World Health Organization (WHO). WHO statement on the second meeting of the IHR emergency committee concerning MERS-CoV. Geneva: WHO; 17 July 2013. Available from: http:// www.who.int/mediacentre/news/statements $/ 2013 /$ mers_ cov_20130717/en/index.html 